\title{
Mechanism of Action of Diallyl Sulphide in Ameliorating the Hematopoietic Radiation Injury
}

\author{
Omika Katoch ${ }^{1}$ Mrinalini Tiwari ${ }^{1} \quad$ Namita Kalra ${ }^{1}$ Paban K. Agrawala ${ }^{1}$ \\ ${ }^{1}$ Department of Radiation Genetics and Epigenetics, Institute of \\ Nuclear Medicine and Allied Sciences, Timarpur, New Delhi, India

\begin{abstract}
Address for correspondence Paban K. Agrawala, PhD, Department of Radiation Genetics and Epigenetics, Institute of Nuclear Medicine and Allied Sciences, Brig SK Mazumdar Road, Timarpur, New Delhi 110054, India (e-mail: paban@inmas.drdo.in).
\end{abstract}

J Health Allied Sci NU 2022;12:57-66.

\begin{abstract}
Keywords

- hematopoietic system

- HDAC inhibitor

- CFU-F

- CFC assay

- COX-2

Diallyl sulphide (DAS), the pungent component of garlic, is known to have several medicinal properties and has recently been shown to have radiomitigative properties. The present study was performed to better understand its mode of action in rendering radiomitigation. Evaluation of the colonogenic ability of hematopoietic progenitor cells (HPCs) on methocult media, proliferation and differentiation of hematopoietic stem cells (HSCs), and transplantation of stem cells were performed. The supporting tissue of HSCs was also evaluated by examining the histology of bone marrow and in vitro colony-forming unit-fibroblast (CFU-F) count. Alterations in the levels of IL-5, IL-6 and COX-2 were studied as a function of radiation or DAS treatment. It was observed that an increase in proliferation and differentiation of hematopoietic stem and progenitor cells occurred by postirradiation DAS administration. It also resulted in increased circulating and bone marrow homing of transplanted stem cells. Enhancement in bone marrow cellularity, CFU-F count, and cytokine IL-5 level were also evident. All those actions of DAS that could possibly add to its radiomitigative potential and can be attributed to its HDAC inhibitory properties, as was observed by the reversal radiation induced increase in histone acetylation.
\end{abstract}

\section{Introduction}

Radiation is used in various fields like power generation, medical diagnostics and therapy, and agricultural and industrial applications. People have become highly dependent on newer technologies and many of them either use or generate some kind of radiations posing the risk of increased exposure. The bad or deleterious effects of radiation are experienced when exposure happens accidentally or incidentally, that is, during occupation, therapy, or in the form of nuclear disaster or explosion, beyond certain level. The magnitude of deleterious effects varies based on quantity and quality of the radiation exposure. In addition, a global threat of nuclear attack also persists, owing to competitive stockpiling

published online May 12, 2021
DOI https://doi.org/ $10.1055 / \mathrm{s}-0041-1730094$ ISSN 2582-4287 of nuclear weapons by several countries. The proliferation of terrorism and asymmetric warfare in 21 st century has rendered the modern world a dangerous place to live and work. The incident of Fukushima catastrophe in Japan and negligent dismantling of a Co-60 $\gamma$-source in New Delhi, India, ${ }^{1}$ are the recent examples of accidental radiation exposure. These incidences, with several others, have increased the attention of researchers toward the development of effective radiation countermeasure modalities. Radiation countermeasure agents provide protection, mitigation, or therapeutic gain against radiation injury. Therapeutic agents are given after the appearance of symptoms to stimulate recovery or repair, while radioprotectors are the agents that are administered prior to radiation exposure to prevent radiation-induced 
cellular and molecular damages, and are useful in cases of planned exposure, radiotherapy, and first responders. Radiomitigating agents are administered during or shortly after radiation exposure but before the onset of clinical symptoms. ${ }^{2}$ Such agents are considered "golden assets" in the events of nuclear emergencies because these agents are useful in case of unplanned exposures and also in the case of mass casualties.

Blood, the major component of hematopoietic system, consists of different types of cells with specialized function providing immunity to an individual. These blood cells have a definitive lifespan and are replenished continuously by the hematopoietic stem cells (HSCs). In humans, HSCs are found in the bone marrow, pelvis, femur, sternum, umbilical cord, and in small numbers in peripheral blood. Hematopoietic system is most sensitive system to radiation damage. Exposure of ionizing radiation like gamma rays to bone marrow leads to the hematopoietic toxicity, disturbances in hematopoietic integrity, destruction of progenitor cells leading to bone marrow failure, hemorrhage, infection, and eventually lethality. ${ }^{3,4}$ In the events of nuclear accidents and most of the radiotherapy cases the probability of exposure to gamma radiation is high. In humans, radiation doses ranging from 1 to $7 \mathrm{~Gy}$ are known to induce hematopoietic syndrome ${ }^{5}$ which consists of symptoms mentioned earlier. Hematopoietic toxicity (reduction of cellular components due to radiation-induced cell death) can be diminished by protecting hematopoietic stem cells as they provide the continuous supply of blood cells (like erythrocyte, granulocyte, monocyte, lymphocyte, etc.). HSCs are capable of self-renewal and have the property to differentiate into different lineage committed progenitor cells (HPCs). Several studies indicate that HDAC inhibitors affect the functioning of stem cells ${ }^{6}$ and the mechanisms for such action are being investigated worldwide.

Diallyl sulphide (DAS) is naturally occurring unique organosulfur compound present in garlic which gives it the pungent flavor. DAS possesses chemopreventive, antimutagenic, antimicrobial, and hepato- and renoprotective properties. $^{7-12}$ It is also a potent modulator of drug-metabolizing enzymes like cytochrome P450. ${ }^{13}$ Several studies have shown that DAS also increase the acetylation of histone proteins possibly thorough inhibition of histone deacetylase enzymes. ${ }^{14}$ Therefore, DAS was considered for evaluation of its radiomitigative potential..$^{15}$ Administration of DAS 2 hours after exposure to lethal whole body gamma radiation in mice rendered $40 \%$ survival, increased the counts of different blood cells and colony-forming units (CFUs) in lethally whole body-irradiated mice.

In the present study, we investigated the efficacy of DAS in mitigating the acute effect of radiation on hematopoietic system in mice model, also known as H-ARS. HDAC inhibitors have been reported to stimulate the proliferation of stem cells in the bone marrow. ${ }^{16,17}$ Effect of DAS on granulocyte colony stimulating factor and COX-2 levels, known to have a role in various pathological and inflammatory scenarios, has been investigated. Study of hematopoietic stem and progenitor cells were performed to evaluate the efficacy of DAS in mitigating hematopoietic system.

\section{Materials and Methods}

\section{Animals}

Animals used in the experiments were 8 to 10 weeks old $\mathrm{C} 57 \mathrm{BL} / 6$ male mice weighing $23.0 \pm 2.0 \mathrm{~g}$. Mice were maintained under controlled conditions of temperature $\left(25 \pm 3^{\circ} \mathrm{C}\right)$, humidity (55\%), and light (12:12 hour of light and dark regimen). The animals were housed in polypropylene cages (not more than 6 mice per cage) and had free access to standard rodent food (Golden Feeds, New Delhi, India) and tap water. Each experimental group consisted of 4 to 6 mice per time point. All experimental procedures had due approval of the institutional animal ethics committee (IAEC).

\section{Irradiation}

Whole body irradiation (WBI) of animals was performed using a Co-60 teletherapy (Bhabhatron-II, GE Health Care) unit at a dose rate of $1 \mathrm{~Gy} / \mathrm{min}$. The animals were restrained in a perforated Perspex box during exposure and without any anesthetics.

\section{Drug Treatment}

DAS (97\% purity; Sigma, St. Louis, Missouri) was diluted in olive oil to attain the desired concentration ( $160 \mathrm{mg} / \mathrm{kg}$ body weight) and administered intraperitoneally in a maximum injection volume of $200 \mu \mathrm{L}$ per animal as reported in our earlier study. ${ }^{15}$

\section{Clonogenic Assay of HPCs}

Mice were treated with DAS (160 mg/kg) 2 hours after irradiating them with $3 \mathrm{~Gy}$ or $5 \mathrm{~Gy}$ since radiation doses beyond this lead to excessive killing of the cells of interest. Mice from different groups were sacrificed 24 hours post treatment. Bone marrow cells from femur were taken out in serum-free media, washed twice with PBS by centrifuging for 10 minutes at $1,000 \mathrm{rpm}$. Cell pellet was collected and resuspended in $0.5 \mathrm{~mL}$ media; $10^{5}$ bone marrow cells were plated on $2 \mathrm{~mL}$ methocult media in $35 \mathrm{~mm}$ culture dishes, incubated at $37^{\circ} \mathrm{C}$ in a carbon dioxide $\left(\mathrm{CO}_{2}\right)$ incubator for 10 to 12 days. Colonies of different progenitors were observed and photographed under light microscope at $10 \times$ magnification. ${ }^{18}$

\section{Histological Examination of Bone Marrow}

Femur bones were fixed with $10 \%$ formalin solution for 24 to 72 hours and decalcified in EDTA for up to 7 to 10 days. After decalcification, the femurs were embedded in paraffin wax and sectioned at a thickness of $5 \mu \mathrm{m}$.

\section{Identification of Hematopoietic Stem Cells}

Mice were irradiated with $3 \mathrm{~Gy}$ where maximum changes in HSC could be observed thoroughly and drug was administered as per the schedule. Twenty-four hours post treatment bone marrow cells were taken out, washed twice with PBS, labeled with respective antibodies, and analyzed for hematopoietic stem cells. HSCs were identified and enumerated according to their surface markers (lin', sca ${ }^{+}, \mathrm{c}^{-\mathrm{kit}^{+}}$cells) using flow cytometer as per the instructions provided with hematopoietic stem and progenitor cell isolation kit (BD cat no 560492). 
The kit contained a lineage cocktail of specific antibodies for CD3 (a marker for T cells), CD45R (B220) (a marker for B cells), Ly6C and Ly6G (Gr1) (a marker for granulocytes), CD11b (Mac1) (a marker for macrophages), and TER-119 (a marker for RBCs). The kit also contained 3 specific antibodies that are used to isolate HPCs and HSCs-CD34 (expressed by HPCs), and Sca- 1 and c-kit (both expressed by HSCs). Briefly, single-cell suspension of bone marrow was washed and $1 \times 10^{6}$ cells were incubated with Fc block for 20 minutes at $4^{\circ} \mathrm{C}$. Then the samples were divided into 3 tubes labeled as unstained, isotype, and sample. Staining the isotype, sample, and the BD CompBeads with FITC CD34, PE c-kit, PE-Cy7 Sca-1 and APC lineage cocktail were done according to the manufacturer's protocol. The samples were incubated in dark for 60 minutes at $4^{\circ} \mathrm{C}$, washed, and resuspended in stain buffer. After adding 1 drop of 7-AAD the cells were acquired on flow cytometer using appropriate filters.

\section{Immunophenotyping of Hematopoietic Progenitors}

Mice were irradiated with $3 \mathrm{~Gy}$ for reasons describes earlier; DAS was administered 2 hours after irradiation. Twenty-four hours post treatment bone marrow cells were taken out from mice of each treated and untreated groups. Cells were washed twice with PBS and then labeled with antibodies against specific surface markers like CD3 for T-cells, B220 for B-cells, Cb11b for monocytes, TER for erythrocytes, and Ly-6G for lymphocytes and analyzed using flow cytometer following the manufacturer's instructions (EBioscience Mouse Hematopoietic lineage Biotin Panel and catalog number 88-7774).

\section{Stem Cell Tracking}

Recipient mice bone marrow was ablated by irradiating at $9 \mathrm{~Gy}$ (which leads to complete bone marrow ablation in the host). Two hours post ablation, $10^{5}$ isolated hematopoietic stem cells from bone marrow of the donor mice were injected intravenously to the recipient mice. Donors were either untreated control mice or drug treated (drug was given 24 hours prior transplantation to the donor mice). Donor's bone marrow hematopoietic stem cells were negatively selected by magnetic cell sorting (MACS) using lineage cell depletion kit (order number 130-090-858) as described by the manufacturer (Milteny Biotech). The isolated lineage negative stem cells $\left(10^{5}\right)$ were stained with CFSE (carboxyfluorescein diacetate succinimidyl ester) and injected to the ablated mice through tail vein. The brief description of MACS is as follow:

The single-cell suspension of donor's bone marrow was centrifuged at $300 \mathrm{~g}$ for 10 minutes, cell pellet was resuspended in $40 \mu \mathrm{L}$ buffer per $10^{7}$ cells, $10 \mu \mathrm{L}$ of biotin-antibody cocktail was added, and it was incubated for 10 minute at 4 to $8^{\circ} \mathrm{C} ; 20 \mu \mathrm{L}$ of antibiotin microbeads were added after adding $30 \mu \mathrm{L}$ buffer provided with the kit and incubated for another 15 minutes at 4 to $8^{\circ} \mathrm{C}$. Cells were then washed and resuspended in $500 \mu \mathrm{L}$ buffer. These magnetically labeled bone marrow cells were then passed through a MACS column which led to the collection of lineage negative stem cells in the elute.

These eluted stem cells were stained with $5 \mu \mathrm{M}$ CFSE dye at $37^{\circ} \mathrm{C}$ for 15 minutes and then injected to the bone marrow ablated recipient mice for their tracking. At different time points (20 minutes to 4 hours) blood, spleen, and bone marrow cells of the recipient mice were taken and were analyzed by flow cytometry for CFSE-stained stem cells of donor mice.

\section{Colony Forming Unit-Fibroblast}

Proliferating capacity of bone marrow tissue including fibroblast, octeoblast, and stromal cells was seen by colonogenic assay (colony-forming unit-fibroblast, CFU-F). Femur was excised 24 hours post treatment. Bone marrow was taken out and washed twice with PBS; $10^{6}, 10^{5}$, and $10^{4}$ nucleated cells were plated per PD 60 in MEM medium and incubated for 12 days. Media was changed every third day. ${ }^{19}$

\section{HDAC Level of Organ Lysate}

Second day post treatment, the liver was taken out, homogenized, and total deacetylated lysine content was estimated using HDAC activity colorimetric assay kit (Biovision, kit name Colorimetric HDAC Activity Assay kit and catalog number K331-100), following the instructions of the manufacturer. Cell lysates containing equal amount of protein from each treatment groups were used after dilution and absorbance was recorded at $405 \mathrm{~nm}$. Total protein in cell lysate was quantified using BCA assay kit (Sigma-Aldrich, catalog number and product code BCA1 and B 9643, respectively) considering BSA as standard. The values were presented as the amount of deacetylated lysine equivalent per microgram of nuclear protein, as estimated from a standard curve prepared using the deacetylated lysine standard provided with the kit.

\section{COX-2 ELISA}

Mice were given different treatments and sacrificed after 24 hours of cervical dislocation. Liver tissue of different groups was dissected out, chopped, homogenized, and tissue extract was analyzed for COX-2 level using mouse cyclooxygenase-2 (TSZ ELISA catalog number M0740) as per the instructions of the manufacturer. Briefly, standards $(0-100 \mathrm{ng} / \mathrm{mL})$ were prepared by serial dilution. Both the standards and samples were incubated in purified mouse COX-2 antibody precoated 96 well plates for 30 minutes at $37^{\circ} \mathrm{C}$. After washing the wells, HRP conjugated enzyme was added and the wells were incubated again for 30 minutes at $37^{\circ} \mathrm{C}$. For color development chromogen A and B substrates were added. Finally, the reaction was stopped by adding stop solution and the yellow color was measured at $450 \mathrm{~nm}$.

\section{Cytokines Level}

The cytokines level was estimated at 48 hours post 2 Gy (which is sufficient to induce deviations in the cytokine levels) using BD CBA plex bead array kit (catalog number 558266) as per the instructions of the manufacturer. This kit employs the single bead population of capture beads with distinct fluorescence intensity and was coated with a capture antibody specific for a soluble protein. The capture bead, PE-conjugated detection reagent, and standard or test samples were incubated together to form sandwich complexes. Following acquisition of samples, data were analyzed using the FCAP Array software. 


\section{Statistics}

Data presented are mean \pm standard deviation (SD) of three independent experiments. Two-way analysis of variance (ANOVA) with Bonferreni test was applied to determine the level of significance and $P<0.05$ was considered significant.

\section{Results}

DAS Administration to Whole Body Irradiated Mice Increased the Colonies of Hematopoietic Stem Cell Supporting Tissue

Bone marrow cells of mice treated with $160 \mathrm{mg} / \mathrm{kg}$ body weight DAS after 2 hours after 5 Gy WBI were more efficient in forming colonies when cultured in vitro as compared with bone marrow cells of mice irradiated with $5 \mathrm{~Gy}$ alone. DAS-treated mice showed increase in CFU-F colonies count ( $26.5 \pm 1.30$ for $5 \mathrm{~Gy}$ alone and $36 \pm 1.75$ for DAS+ $5 \mathrm{~Gy}$, i.e., nearly 1.5 -fold). In DAS alone-treated mice the number of colonies was $45.5 \pm 2.25$ compared with $50.34 \pm 2.51$ colonies in control mice (-Fig. 1).

\section{Administration of DAS to Whole Body Irradiated Mice Protected the Microarchitecture of Bone Marrow}

At 84 hours post irradiation (5 Gy WBI), the bone marrow cellularity (number of viable cells) was severely damaged. It significantly reduced the marrow cellularity as compared with control group. The average number of nucleated cells per unit area $(35 \times 35 \mu \mathrm{m})^{2}$ was counted. The average count of nucleated cells for control mice was $37.5 \pm 1.80$, while in $5 \mathrm{~Gy}$ irradiated group the count was $2.5 \pm 0.12$ (i.e., nearly 15 -fold reduction). In irradiated group, the enucleated cells (red blood cells [RBCs]) were more, with the average number per unit area $33 \pm 1.65$, compared with $5.5 \pm 0.27$ (control group, nearly 6 -fold change). Treatment of irradiated mice with DAS after 2 hours of exposure reduced this effect of radiation by increasing the cellularity of bone marrow. The enucleated

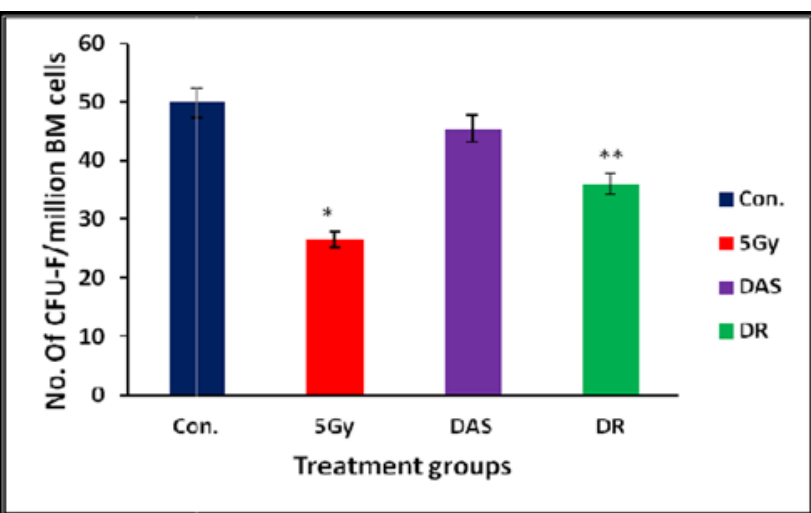

Fig. 1 CFU-F indicates the hematopoietic stem cell supporting tissue. Mice were irradiated with 5 Gy and DAS was administered 2 hours post irradiation of $5 \mathrm{~Gy}$. After 24 hours of $5 \mathrm{~Gy}$, bone marrow cells were taken out and $1 \times 10^{5}$ cells were cultured for 12 days. Treatment of irradiated mice with DAS significantly increased the number of CFU-F colonies as compared with irradiation group alone. Here ${ }^{*}$ indicates significant value compared with control while ** indicates significant compared with radiation; $p<0.05$. cells (RBCs) appeared in pockets and their number also decreased as compared with radiation alone group. The average count of nucleated cells per unit area was $39 \pm 1.92$ and that of enucleated cells was $7.5 \pm 0.35$. DAS alone showed increased number of nucleated cells. The average number of nucleated and enucleated cells in DAS-treated mice bone marrow was $49.75 \pm 2.40$ and $3 \pm 0.15$, respectively ( - Fig. 2 ).

\section{Immunophenotyping of Hematopoietic Progenitors}

\section{DAS Administration to Whole Body Irradiated Mice Increased the Number of Hematopoietic Stem and Lineage Progenitor Cells}

Immunophenotyping of hematopoietic progenitors showed that DAS protected different lineage progenitor cells. Exposure of mice to $3 \mathrm{~Gy}$ WBI (a dose sufficient to induce visible changes in HSCs) decreased the number of different lineage progenitors except TER positive cells. The lymphocyte progenitors were most affected by radiation exposure while erythrocyte progenitors were resistant. The fraction of different progenitors decreased by radiation exposure was as- CD3-positive progenitors decreased from control values of $1.9 \pm 0.09$ to $0.133 \pm 0.006$ ( $\sim 15$-fold), B220-positive progenitors decreased from $39.1 \pm 1.95$ (control value) to $4.766 \pm 0.23$ ( 8.3-fold), Cb11b-positive decreased from $52.3 \pm 2.56$ (control value) to $23.8 \pm 1.19$ ( 2.25-fold), and Ly-6G-positive decreased from $36 \pm 1.3$ (control value) to $1.766 \pm 0.082$ ( 21-fold). DAS administration in irradiated mice reduced this effect of radiation and increased the number of different lineage progenitor cells count (CD3+ to $4.83 \pm 0.24, \mathrm{~B} 220+$ to $12.66 \pm 0.63$, $\mathrm{Cb} 11 \mathrm{~b}+$ to $84.6 \pm 4.23$, and $\mathrm{Ly}-6 \mathrm{G}+$ to $66.9 \pm 3.34)$ except TER+ (1.8 \pm 0.09$)$. However, erythrocyte progenitors showed amplification in irradiated mice bone marrow $(10.46 \pm 0.52)$ as compared with control ones $(1 \pm 0.05)$. DAS alone showed no significant change in different fractions of progenitors (CD3+ to $2.73 \pm 0.13, \mathrm{~B} 220+$ to $33.9 \pm 1.65, \mathrm{Cb} 11 \mathrm{~b}+$ to $56.5 \pm 2.80$, Ly- $6 \mathrm{G}+$ to $37.5 \pm 1.81$, and TER+ to $1.9 \pm 0.09$ ). The sequence

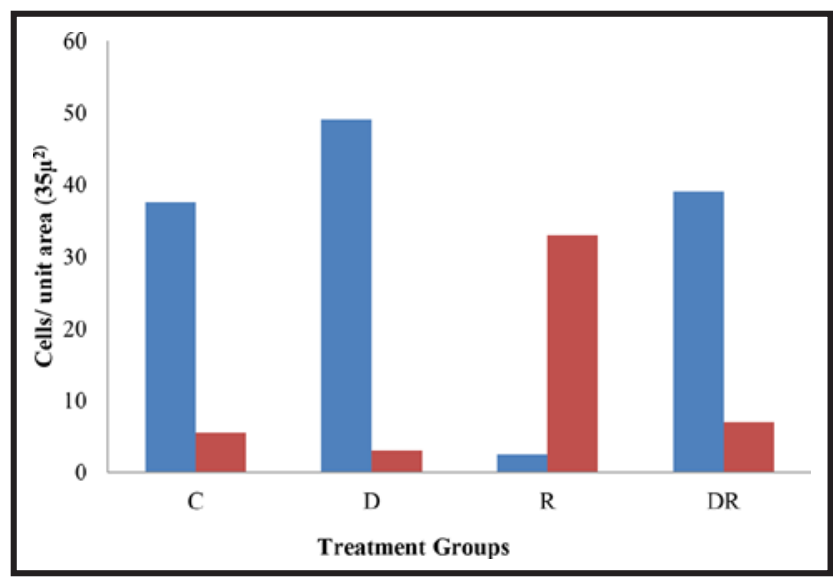

Fig. 2 Bone marrow sections were prepared at 84 hours post 5 Gy, sections were stained with H\&E stain, and number of nucleated and enucleated cells was counted microscope and presented as nucleated (blue) versus enucleated (red) cells per unit area. 
of different hematopoietic progenitors affected by radiation exposure in decreasing order was as follows: lymphocyte progenitors $($ Ly-6G) $>$ T cell progenitors $($ CD3 $)>$ B cell progenitors (B220) > monocyte progenitors (Cb11b) > erythrocyte progenitors (TER) ( - Fig. 3).

\section{Hematopoietic Stem Cell Enumeration}

Hematopoietic stem cells and progenitors are most radiosensitive cells in the bone marrow. Radiation exposure (3 Gy WBI) significantly decreased the stem cell count to $737.23 \pm$ 36.05 from $5198.01 \pm 250.98$ (count of stem cells in control mice bone marrow), which was 7-fold decreased in stem cells as compared with control mice. Mice treated with DAS after 2 hours of radiation exposure showed protection of stem cells from radiation. DAS-treated irradiated mice showed increase in the stem cell count, $3332.64 \pm 166.63$, as compared with radiation alone, $737.23 \pm 36.86$ ( $\sim 4.5$-fold). DAS alone had no significant change; the count of hematopoietic stem cells per million bone marrow cells was $4668 \pm 233.4$ (-Fig. 4).

\section{DAS Administration Increased the Colonies of Different Hematopoietic Progenitor Cells}

Radiation reduced the average colony count of different progenitor cells in culture with both $3 \mathrm{~Gy}$ and $5 \mathrm{~Gy}$ doses of radiation as compared with control ones (-Table 1). The 3 Gy and $5 \mathrm{~Gy}$ doses reduced the number of CFU-GM colonies from $9 \pm 0.38$ (control value) to $2 \pm 0.1$ ( 4 .5-fold) and $1 \pm 0.2$ ( 9-fold), CFU-GEMM from $86 \pm 3.5$ (control value) to 0 and $1 \pm 0.46$ ( 85-fold), CFU-E from $3 \pm 0.12$ (control value) to 1 \pm 0.05 and $1 \pm 0.04$ ( $\sim$-fold), and BFU-E from $55 \pm 2.61$ (control value) to $9 \pm 0.45$ ( 6 -fold) and $10 \pm 0.5$ ( $~ 5$ fold), respectively. Progenitor cells of mice treated with DAS after 2 hours of radiation exposure produced more number of colonies. However, in 3 Gy exposed mice DAS restored the progenitor's

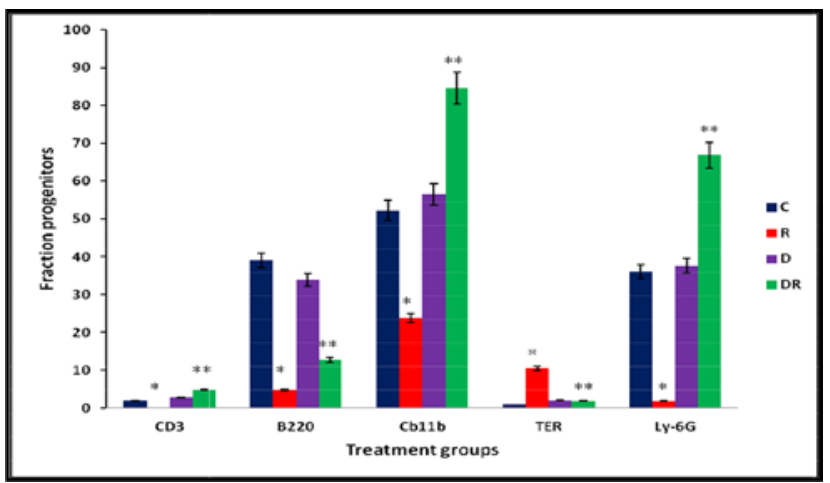

Fig. 3 Number of different hematopoietic lineage progenitors was measured in mice bone marrow. Mice were irradiated with $3 \mathrm{~Gy}$. DAS was given to irradiated mice post 2 hours. Different lineage progenitors were evaluated after 24 hours of 3 Gy by flow cytometry using labeled antibodies against specific surface markers (CD3 for T cells, B220 for B cells, Cb11b for monocytes, TER for erythrocytes, and Ly-6G for lymphocytes). DAS treatment significantly increased the fraction of different lineage progenitors except erythroid progenitors in irradiated mice. Here ${ }^{*}$ indicates significant value compared with control while ${ }^{* *}$ indicates significant compared with radiation; $p<0.05$.

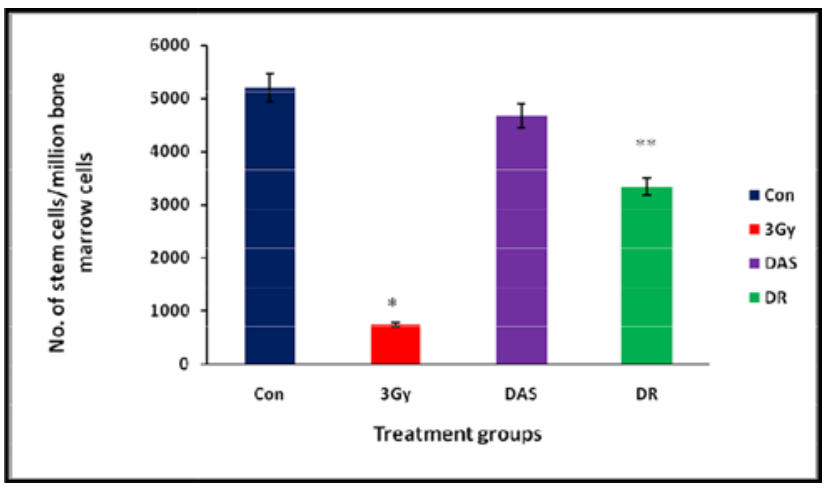

Fig. 4 Mice were irradiated with 3 Gy and after 2 hours DAS was given. Hematopoietic stem cells were evaluated 24 hours post 3 Gy by flow cytometry using hematopoietic stem and progenitor cell isolation kit that contained a lineage cocktail of specific antibodies labeled with a fluorochrome. DAS administration significantly increased the hematopoietic stem cell count in irradiated mice as compared with irradiated mice alone. Here * indicates significant value compared with control while ${ }^{* *}$ indicates significant compared with radiation; $p<0.05$.

colonogenic property to a higher extent in comparison to 5 Gy exposed ones ( - Table 1, - Fig. 5a, b).

\section{Tracking of Hematopoietic Stem Cells}

Recipient mice receiving bone marrow from DAS-treated donor mice (DAS donor) showed significant increased count of average number of CFSE stained cells in different target organs (blood, bone marrow, and spleen) at all time points studied (except in bone marrow and spleen at 20 minutes, and in blood at 3 hours post transplantation) compared with those receiving bone marrow from donor mice without DAS treatment (control donor).

Blood of mice receiving bone marrow from DAS donor mice showed increased CFSE-stained cells compared with control donor at all time points except at 3 hours. The average number of CFSE-stained cells in the blood of recipient mice (receiving transplantation from DAS donor vs. control donor) was higher (among other organs studied) at 2 and 4 hours ( $11.03 \pm 0.46$ vs. $1.90 \pm 0.1,15.04 \pm 0.75$ vs. $0.17 \pm$ 0.008 , respectively). At 20 minutes, 30 minutes, and 1 hour, the average number of CFSE-stained cells from DAS donor versus control donor were $2.18 \pm 0.10$ versus $0.88 \pm 0.04,9.28$ \pm 0.46 versus $2.49 \pm 0.12$, and $7.2 \pm 0.36$ versus $2.4 \pm 0.12$. At 3 hours post bone marrow transplantation, the average number of CFSE-stained cells were $7.4 \pm 0.37$ (DAS donor) versus $11.2 \pm 0.48$ (control donor).

Bone marrow of mice receiving transplantation from DAS donor mice showed increased CFSE-stained cells compared with control donor at all time points except at 20 minutes. The number was higher at 2 and 4 hours post transplantation. The average number of CFSE-stained cells from DAS donor versus control donor were $28.6 \pm 1.28,18.2 \pm 0.84,1.97$ $\pm 0.09,27.6 \pm 1.30$, and $1.37 \pm 0.05$ versus $12.7 \pm 0.60,18 \pm$ $0.82,0,22.8 \pm 1.06$, and $0.083 \pm 0.004$ at 30 minutes, 1 hour, 2 hours, 3 hours, and 4 hours, respectively. At 20 minutes the count in DAS donor was $2.56 \pm 0.13$ versus $32.85 \pm 1.54$ (control donor). 
Table 1 The average number of colonies of different hematopoietic progenitor cells in vitro

\begin{tabular}{|l|l|l|l|l|}
\hline & CFU-GEMM & BFU-E & CFU-GM & CFU-E \\
\hline Con & 9 & 55 & 86 & 3 \\
\hline DAS & 15 & 50 & 82 & 2 \\
\hline 3 Gy & 2 & 9 & 0 & 1 \\
\hline 5 Gy & 1 & 10 & 1 & 1 \\
\hline DAS + 3 Gy & 8 & 43 & 55 & 8 \\
\hline DAS + 5 Gy & 3 & 4 & 5 & 0 \\
\hline
\end{tabular}

Abbreviations: DAS, diallyl sulphide.
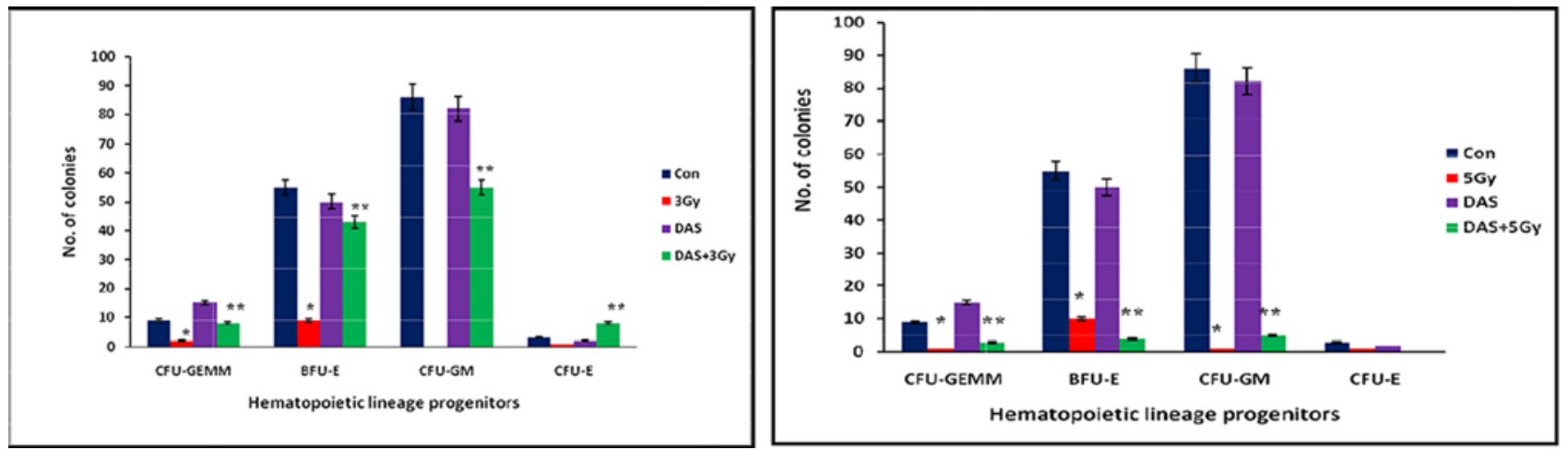

Fig. 5 (a) The proliferative ability of lineage progenitors was evaluated by CFC assay. Bone marrow of irradiated mice was taken out after 24 hours of 3 Gy and $1 \times 10^{5}$ nucleated cells were cultured in methocult media for 12 days to form colonies. Colonies were counted under microscope at 10x magnification. DAS treatment significantly increased the number of colonies of different progenitors in 3 Gy irradiated mice. Here ${ }^{*}$ indicates significant value compared with control while ${ }^{* *}$ indicates significant compared with radiation; $p<0.05$. (b) The proliferative ability of lineage progenitors was evaluated by CFC assay. Bone marrow of irradiated mice was taken out after 24 hours of 5 Gy and $1 \times$ $10^{5}$ nucleated cells were cultured in methocult media for 12 days to form colonies. Colonies were counted under microscope at $10 \times$ magnification. DAS treatment significantly increased the number of colonies of different progenitors except BFU-E and CFU-E in 5 Gy irradiated mice. Here ${ }^{*}$ indicates significant value compared with control while ${ }^{* *}$ indicates significant compared with radiation; $p<0.05$.

Spleen of recipient mice also showed the same trend as shown by bone marrow; it showed increased average CFSE count at all time points except at 20 minutes. The number was higher at 1 and 3 hours post transplantation. The average number of CFSE-stained cells from DAS donor versus control donor were $26.9 \pm 1.25,51.5 \pm 2.0,0.39 \pm 0.01,70.9 \pm 3.0$, and $1.4 \pm 0.07$ versus $17.2 \pm 0.80,18.1 \pm 0.85,0,22.1 \pm 1.05$, and $0.090 \pm 0.004$ at 30 minutes, 1 hour, 2 hours, 3 hours, and 4 hours, respectively. At 20 minutes the count in DAS donor

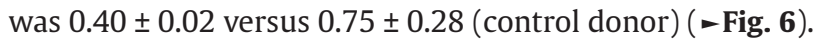

\section{DAS Administration Suppressed the Expression of COX-2}

Irradiated mice showed increased COX-2 level (104.366 \pm 5.10$)$ in liver extract after 24 hours of irradiation as compared with control group $(60.03 \pm 3)$. DAS administration to irradiated mice reduced this effect of radiation by decreasing the COX 2 level to $72.10 \pm 3.32$. DAS alone had shown no significant effect $(66.43 \pm 3.21)$ as compared with control mice. In other words DAS was observed to act as a COX-2 inhibitor ( - Fig. 7).

\section{Cytokines Level}

Irradiation of mice with $2 \mathrm{~Gy}$ significantly increased the level of IL-6 in mice plasma. Irradiated mice plasma showed 16-fold
$(8,661 \mathrm{pg} / \mathrm{mL})$ increased IL-6 level as compared with control mice ( $546 \mathrm{pg} / \mathrm{mL}$ ). DAS treatment after 2 hours of $2 \mathrm{~Gy} \mathrm{WBI}$ reduced IL-6 level from 8,661 to $1542.96 \mathrm{pg} / \mathrm{mL}$ (or 5.6-fold) in irradiated mice. DAS alone also showed an increased level of IL-6 $(1,533 \mathrm{pg} / \mathrm{mL})$ as compared with control values $(546 \mathrm{pg} / \mathrm{mL})$ (-Fig. 8).

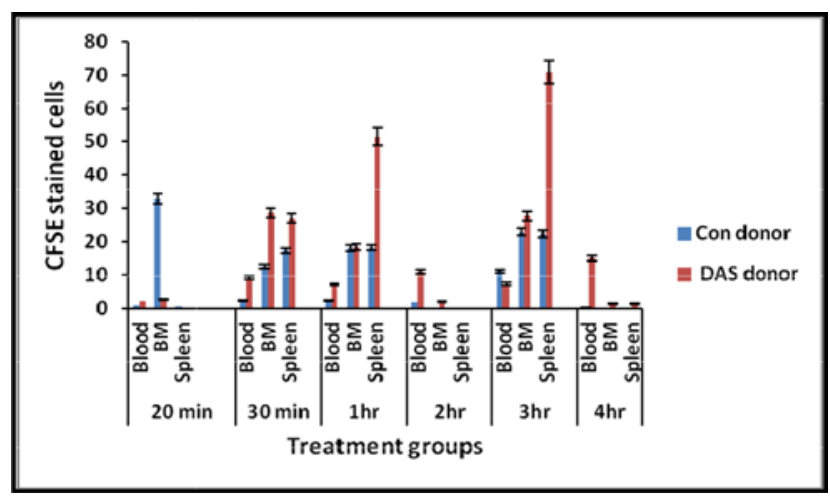

Fig. 6 Recipient mice were irradiated with 9 Gy to ablate bone marrow cells. Bone marrow was transplanted from the mice treated with or without DAS. At designated time, that is, 20 minutes, 30 minutes, 1 hour, 2 hours, 3 hours, and 4 hours post transplantation different samples were analyzed for CSFE stain. Mice receiving bone marrow form DAS donor showed significant high number of CFSE-stained cell in all the samples (blood, bone marrow, and spleen) at all time points except in bone marrow and spleen at 20 minutes, and in blood at 3 hours. 
Mice irradiated with 2 Gy showed opposite effect on IL-5 level as compared with IL-6 level. Irradiation significantly increased the IL-5 level from 37 (control mice value) to $55 \mathrm{pg} / \mathrm{mL}$ in mice plasma. DAS-treated WBI-irradiated mice showed increased level $(534 \mathrm{pg} / \mathrm{mL})$ that was significantly higher as compared with both control and irradiated mice values. DAS alone also increased the level to $148.6 \mathrm{pg} / \mathrm{mL}$ ( - Fig. 9).

\section{HDAC Level Was Reduced by DAS Administration}

Histone deacetylase level was assessed at 24 hours post irradiation and the values were presented as the amount of deacetylated lysine equivalent per microgram nuclear protein. The total deacetylated lysine content in the liver tissue increased following radiation exposure; 5 Gy irradiation increased the total deacetylated lysine from $33.84 \pm 1.02$ (control value) to $57.63 \pm 2.08$ ( $~ 1.7$ times). DAS administration after 2 hours of $5 \mathrm{~Gy}$ exposure reduced the total deacetylated lysine to $38.75 \pm 1.65$. However, DAS alone-treated mice showed a slight decreased total decaetyaled lysine (30.12 \pm 1.25) as compared with control mice (-Fig. 10).

\section{Discussion}

Hematopoietic system failure appears to be the major cause of death in the scenario of chemotherapy or radiation exposure. Our results from this study are consistent with the earlier observations indicating HDAC inhibitors enhance the self-renewal of bone marrow hematopoietic stem cells. ${ }^{16,17}$ Several studies have already shown that the protection of hematopoietic colony-forming cells lead to increased survival in mice against lethal irradiation when anti-inflammatory and immunoenhancing agents were given after irradiation. . $^{1020-22}$ Our earlier study showed 37\% survival in mice against lethal WBI, which seems to be attributed by the protective action of DAS on hematopoietic system. ${ }^{15}$ DAS administration increased the count of CFU-s that shows the colonogenic capacity of hematopoietic stem and progenitor cells in the form of visible colonies on spleen and further

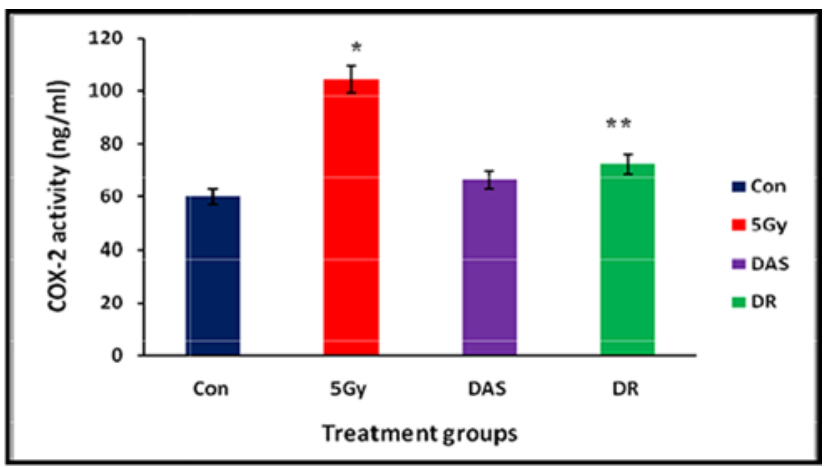

Fig. 7 COX-2 activity was measured in liver extract, 24 hours post irradiating mice with 5 Gy using ELISA kit. Absorbance was taken at $450 \mathrm{~nm}$. DAS significantly decreased the activity of COX-2 in irradiated mice liver extract. Here ${ }^{*}$ indicates significant value compared with control while ${ }^{* *}$ indicates significant compared with radiation; $p<0.05$. restored the WBCs and lymphocyte count on one hand, while on the other hand decreased the percentage of apoptotic cells in bone marrow of irradiated mice. ${ }^{15}$

Severity of radiation on hematopoietic system can be assessed by bone marrow cellularity. Histology of the femur bone is the most preferred method to assess cellularity. In this study, DAS administration to sublethally irradiated mice significantly increased bone marrow cellularity. These data are supported by our previous data of apoptosis ${ }^{15}$ where a reduction of bone marrow cell death as a result of DAS administration is reported. Effect of DAS treatment on the radioresistant RBCs count at the expense of other blood cells was evident in histological sections. The same result was found when a fraction of different progenitors in the bone marrow was evaluated. These results are in accordance with our previous hematological data showing more RBCs in irradiated mice than other blood cell types. Administration of DAS restored the other blood cells to the control level. DAS also improved the morphology of bone and increased the number of nucleated cells in bone marrow of irradiated mice. Improvement of hematopoietic microenvironment promotes hematopoietic stem cell and hematopoietic progenitor cell proliferation and differentiation.

Stem cell supporting tissue protection was also reported in our study. Bone marrow microenvironment termed as "niche" supports the hematopoietic stem cells in their growth, proliferation, and normal functioning. Hematopoietic stem cells proliferate and differentiate to different progenitors within this niche. Radiation exposure causes damage to this microenvironment by causing death of the stem cells as well as the supporting cells. Stromal fibroblast, which is the main component of hematopoietic microenvironment, supports the differentiation and proliferation of blood cells by providing the structural support for hematopoietic cell growth as well as secreting various HGF to stimulate hematopoiesis. ${ }^{23}$ The results of this study demonstrated the beneficial effects of DAS on hematopoietic microenvironment by enhancing

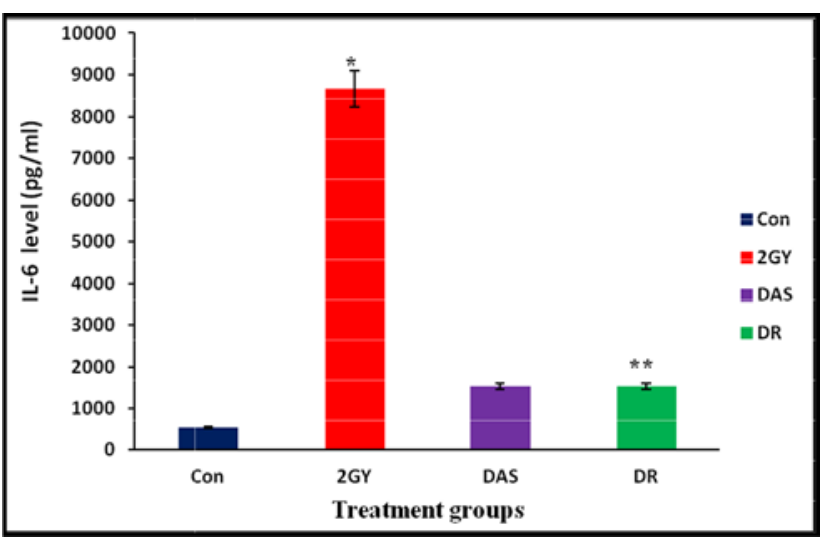

Fig. 8 Mice were exposed whole body to 2 Gy; DAS was administered 2 hours post irradiation. Blood plasma was taken out after 48 hours of radiation and IL- 6 level was evaluated using BD CBA plex bead array kit. DAS-treated mice showed decreased IL- 6 level in their plasma as compared to irradiated mice. Here ${ }^{*}$ indicates significant value compared with control while ** indicates significant compared with radiation; $p<0.05$. 


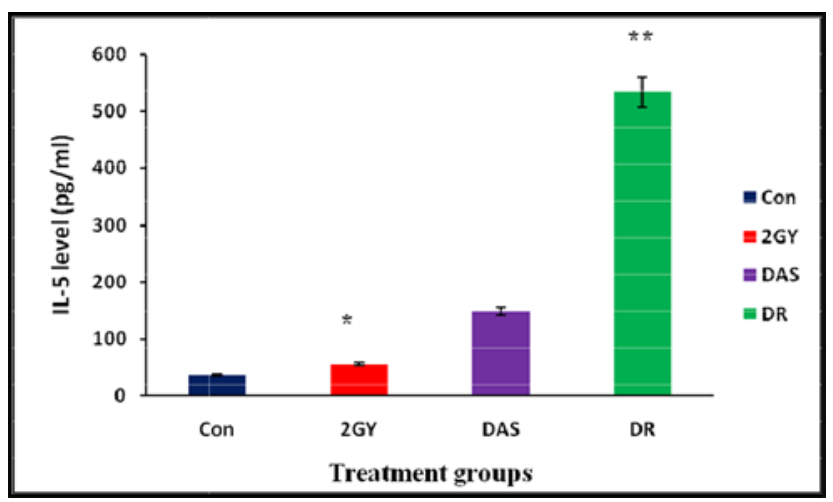

Fig. 9 Mice were exposed whole body to 2 Gy; DAS was administered 2 hours post irradiation. Blood plasma was taken out after 48 hours of radiation and IL-5 level was evaluated. DAS-treated mice showed increased IL-5 level in their plasma as compared irradiated mice. Here ${ }^{*}$ indicates significant value compared with control while ${ }^{* *}$ indicates significant compared with radiation; $p<0.05$.

the CFU-F colony formation. DAS treatment in irradiated mice doubled the CFU-F count as compared with irradiated mice alone.

Increase in CFU-F colonies count in DAS-treated mice helps the stem cells in their proper function, proliferation, and differentiation as evident from the flow cytometric data of hematopoietic stem and progenitor cells. Increase in the percentage or count of hematopoietic stem cells provides the continuous supply of different lineage progenitors. Hematopoietic stem cells are lin', c-kit', and $\mathrm{sca}^{+}$. Identification and enumeration of hematopoietic stem and progenitor cells was done by flowcytometry. Also, when cultured on semisolid (methocult) media containing appropriate growth factors and other supplements, hematopoietic progenitor cells (HPCs) from DAS-treated mice proliferate and formed more colonies (colony-forming cell or CFC assay) than that of irradiated mice alone.

Staining or labeling of stem cells from donor mice with CFSE, and then transplanting them to whole body lethally irradiated mice (ablated bone marrow) is considered an effective method to track stem cells. Recipient mice receiving stem cells from DAS-treated mice showed increased CFSE-stained stem cell counts in all target organs studied at all time points as compared with those receiving bone marrow from control or untreated mice. The CFSE-stained stem cells from DAS donor were higher in bone marrow (and not in spleen or blood) of recipient mice at 30 minutes post transplantation; in blood sample it was higher at 4 hours and in spleen sample of recipient mice it was higher at 3 hours post transplantation.

Different cytokines also promote the proliferation and differentiation of HSCs. In our study, we found that DAS increased the level of IL-5 cytokine in blood plasma. IL-5 is a specific hematopoietic growth factor that promotes the growth and differentiation of eosinophils (one type of WBCs). B chain of IL-5 shares the similar signaling pathway like that of GM-CSF and IL-3 which are the well known factors able to stimulate proliferation of eosinophil. ${ }^{24}$ IL-5 promotes the growth and differentiation of immature

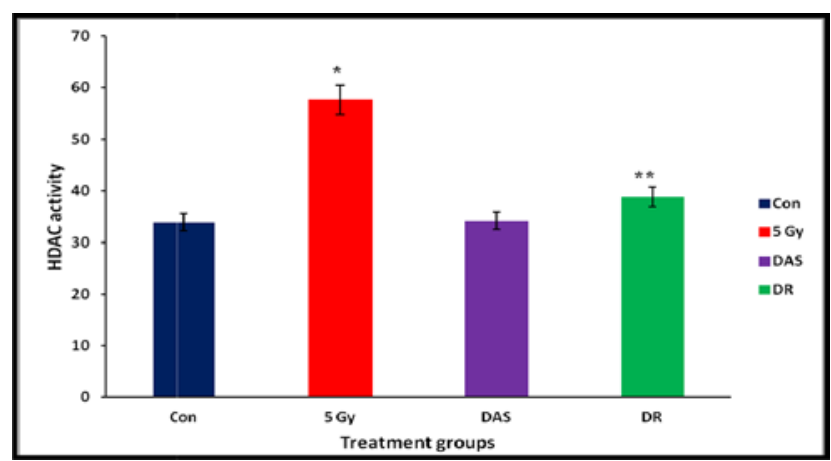

Fig. 10 Mice were irradiated with 5 Gy; DAS was administered after 2 hours. Total deacetylated lysine content was measured by using HDAC activity colorimetric assay kit 24 hours post irradiation. The absorbance was taken at $405 \mathrm{~nm}$. Treatment of DAS 2 hours post 5 Gy significantly reduced the deacetylated lysine in liver extract of irradiated mice. Here ${ }^{*}$ indicates significant value compared with control while ${ }^{* *}$ indicates significant compared with radiation; $p<0.05$.

erythroid progenitors BFU-E but differentiation of CFU-E is inhibited by IL-5. It strongly stimulates the proliferation, activation, and differentiation of eosinophils. These data also provide evidence indicating DAS-mediated increase in WBCs count in blood.

Interleukin-6 (IL-6) is a pleiotropic cytokine. It plays a central role in host defense against environmental stress such as infection and injury. IL-6 has been implicated in the generation and propagation of chronic inflammation. Under physiological conditions IL-6 is barely detectable but during inflammation its level raises and it plays a significant role in driving chronic inflammation, autoimmunity, endothelial cell dysfunction, and fibrogenesis. IL-6 is also a profibrogenic cytokine. But it has been shown to increase or decrease fibroblast proliferation, increase fibroblast collagen, glycosaminoglycan, tissue inhibitor of metalloproteinases-1 (TIMP-1) synthesis, and increase MCP-1. In systemic sclerosis patients, IL-6 induces excess of vascular endothelial growth factor (VEGF), which causes increased angiogenesis and vascular permeability. Moreover, in bone marrow IL-6 activates osteoclasts leading to bone resorption and osteoporosis. TSA, another well-studied HDAC inhibitor, was found to reduce the expression of OA-associated proteins MMP1, MMP3, and MMP13 and proinflammatory cytokines TNF- $\alpha$, IL- $1 \beta$, and IL- $6 .{ }^{25}$ One study found that the proinflammatory cytokines such as IL- $1 \beta$ and IL- 6 induced the overexpression of COX-2 in colorectal cancer. ${ }^{26}$ In our study, DAS administration reduced the level of IL- 6 in irradiated mice that leads to decrease in COX-2 level. Both proinflammatory cytokine IL-6 and COX-2 are associated with fibrosis in later stages of damage or infection caused by radiation. Elango et al, in 2004, also found inhibition of COX-2 by DAS in HEK 293T cells. ${ }^{27}$

Bo et al, in $2012,^{28}$ showed that DAS had the capacity of inhibiting the histone deacetylase which helps in prevention of cancer by inducing histone hyperacetylation and by reactivating tumor suppressor genes that are epigenetically silenced. ${ }^{28}$ - Fig. 11 shows various actions of DAS on hematopoietic system of mice which cumulative could 


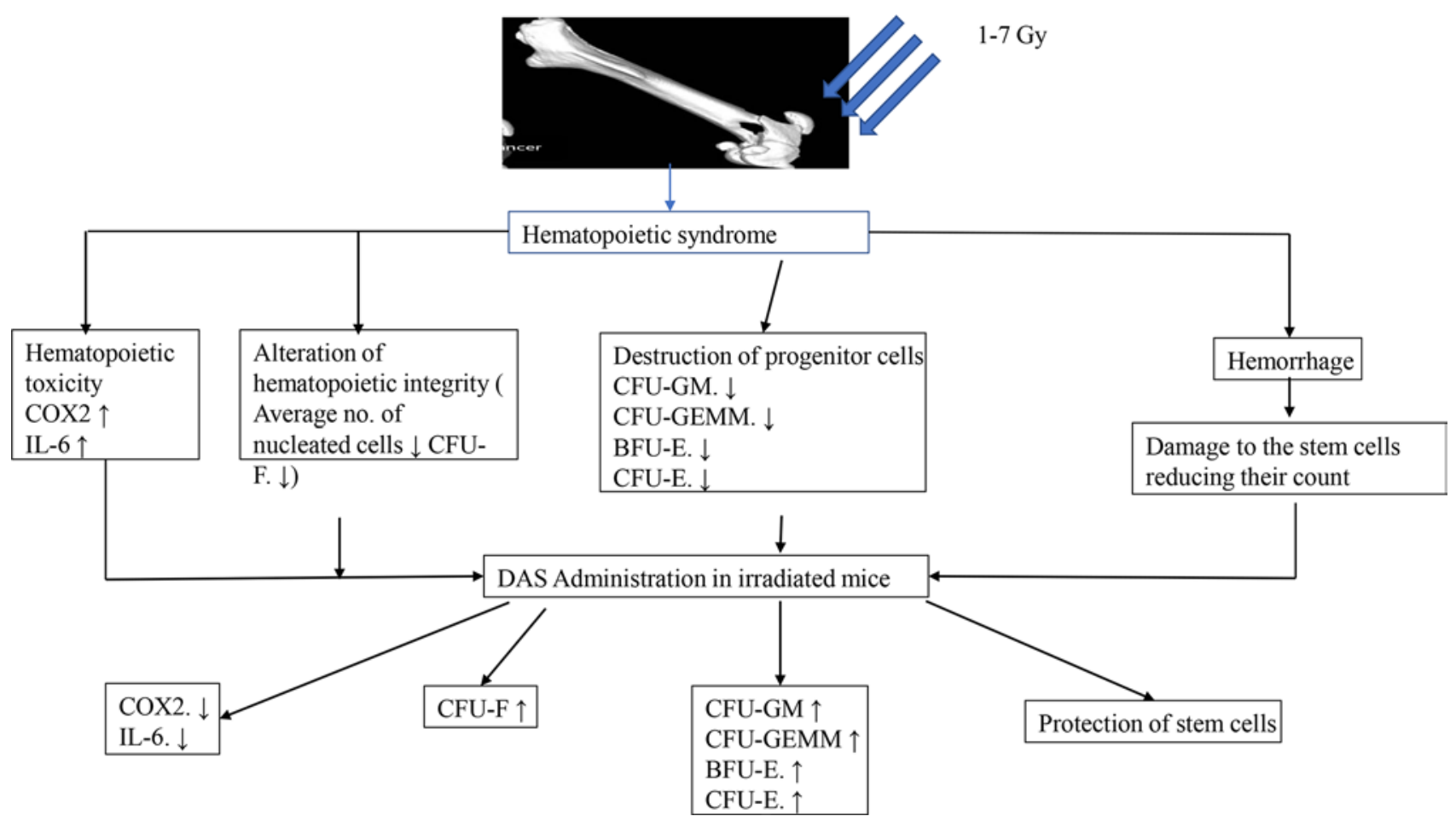

Fig. 11 Schematic presentation of DAS-mediated mitigation of radiation injury.

mitigate radiation injury. By in vitro study, they also found the increased histones $\mathrm{H} 3$ and $\mathrm{H} 4$ acetylation in human gastric cancer cells. This property of DAS might be its mode of action in this study because it significantly inhibits the HDAC activity in liver tissue extract of irradiated mice.

\section{Conflict of Interest}

None declared.

\section{References}

1 Katoch O, Kumar A, Adhikari JS, Dwarakanath BS, Agrawala PK. Sulforaphane mitigates genotoxicity induced by radiation and anticancer drugs in human lymphocytes. Mutat Res 2013;758(1-2):29-34

2 Katoch O, Dwarakanath BS, Agrawala PK. HDAC inhibitors: applications in oncology and beyond. HOAJ Biol 2013;2:2

3 Green DE, Rubin CT. Consequences of irradiation on bone and marrow phenotypes, and its relation to disruption of hematopoietic precursors. Bone 2014;63:87-94

4 Shao L, Luo Y, Zhou D. Hematopoietic stem cell injury induced by ionizing radiation. Antioxid Redox Signal 2014; 20(9):1447-1462

5 Xiao M, Whitnall MH. Pharmacological countermeasures for the acute radiation syndrome. Curr Mol Pharmacol 2009; 2(1):122-133

6 Kretsovali A, Hadjimichael C, Charmpilas N. Histone deacetylase inhibitors in cell pluripotency, differentiation, and reprogramming. Stem Cells Int 2012;2012:184154

7 Singh VK, Ducey EJ, Brown DS, Whitnall MH. A review of radiation countermeasure work ongoing at the Armed Forces Radiobiology Research Institute. Int J Radiat Biol 2012; 88(4):296-310

8 Landauer MR, Castro CA, Benson KA, Hogan JB, Weiss JF. Radioprotective and locomotor responses of mice treated with nimodipine alone and in combination with WR-151327. J Appl Toxicol 2001;21(1):25-31
9 Vrinda B, Uma Devi P. Radiation protection of human lymphocyte chromosomes in vitro by orientin and vicenin. Mutat Res 2001;498(1-2):39-46

10 Nair CKK, Parida DK, Nomura T. Radioprotectors in radiotherapy. J Radiat Res (Tokyo) 2001;42(1):21-37

11 Goel HC, Prasad J, Singh S, et al. Radioprotective potential of an herbal extract of Tinospora cordifolia. J Radiat Res (Tokyo) 2004;45(1):61-68

12 C Jagetia G. Radioprotective potential of plants and herbs against the effects of ionizing radiation. J Clin Biochem Nutr 2007;40(2):74-81

13 Kim SH, Lee IC, Baek HS, et al. Induction of cytochrome P450 3A1 expression by diallyl disulfide: protective effects against cyclophosphamide-induced embryo-fetal developmental toxicity. Food Chem Toxicol 2014;69:312-319

14 Brown SL, Kolozsvary A, Liu J, Ryu S, Kim JH. Histone deacetylase inhibitors protect against and mitigate the lethality of total-body irradiation in mice. Radiat Res 2008;169(4):474-478

15 Katoch O, Khan GA, Dwarakanath BS, Agrawala PK. Mitigation of hematopoietic radiation injury by diallyl sulphide. J Environ Pathol Toxicol Oncol 2012;31(4):357-365

16 Young JC, Wu S, Hansteen G, et al. Inhibitors of histone deacetylases promote hematopoietic stem cell self-renewal. Cytotherapy 2004;6(4):328-336

17 De Felice L, Tatarelli C, Mascolo MG, et al. Histone deacetylase inhibitor valproic acid enhances the cytokine-induced expansion of human hematopoietic stem cells. Cancer Res 2005;65(4):1505-1513

$18 \mathrm{Xu} \mathrm{MJ}$, Tsuji K, Ueda T, et al. Stimulation of mouse and human primitive hematopoiesis by murine embryonic aorta-gonad-mesonephros-derived stromal cell lines. Blood 1998;92(6):2032-2040

19 Hofer EL, Labovsky V, La Russa V, et al. Mesenchymal stromal cells, colony-forming unit fibroblasts, from bone marrow of untreated advanced breast and lung cancer patients suppress fibroblast colony formation from healthy marrow. Stem Cells Dev 2010;19(3):359-370 
20 Butorin I, Cherdyntseva N, Averin S. Radioprotective effect of extract from root of Glycyrrhiza is associated with its ability to stimulate the bone marrow hemopoiesis. In: International Symposium on Radiation and Hemopoiesis 2001, Satellite Symposium: Radioprotectors for Human Health, p 17, Abstract Book, July 2001, Kyoto, Japan

21 Van der Meeren A, Mouthon MA, Vandamme M, Squiban C, Aigueperse J. Combinations of cytokines promote survival of mice and limit acute radiation damage in concert with amelioration of vascular damage. Radiat Res 2004;161(5):549-559

22 Farese AM, Williams DE, Seiler FR, MacVittie TJ. Combination protocols of cytokine therapy with interleukin-3 and granulocyte-macrophage colony-stimulating factor in a primate model of radiation-induced marrow aplasia. Blood 1993;82(10):3012-3018

23 Liu M, Tan H, Zhang X, et al. Hematopoietic effects and mechanisms of Fufang éjiao jiang on radiotherapy and chemotherapy-induced myelosuppressed mice. J Ethnopharmacol 2014;152(3):575-584
24 Broughton SE, Nero TL, Dhagat U, et al. The $\beta c$ receptor family-structural insights and their functional implications. Cytokine 2015;74(2):247-258

25 Cai D, Yin S, Yang J, Jiang Q, Cao W. Histone deacetylase inhibition activates Nrf2 and protects against osteoarthritis. Arthritis Res Ther 2015;17:269

26 Maihöfner C, Charalambous MP, Bhambra U, Lightfoot T, Geisslinger G, Gooderham NJ; Colorectal Cancer Group. Expression of cyclooxygenase-2 parallels expression of interleukin-1beta, interleukin-6 and NF-kappaB in human colorectal cancer. Carcinogenesis 2003;24(4):665-671

27 Elango EM, Asita H, Nidhi G, Seema P, Banerji A, Kuriakose MA. Inhibition of cyclooxygenase-2 by diallyl sulfides (DAS) in HEK 293T cells. J Appl Genet 2004;45(4):469-471

28 Su B, Shu LX, Su J, et al. Diallyl disulfide increases histone acetylation and P21WAF1 expression in human gastric cancer cells in vivo and in vitro. Biochem Pharmacol 2012;1:106 